\title{
ANÁLISE DA RELAÇÃO ENTRE OS PARÂMETROS PETROFÍ́SICOS E AS CURVAS DE FASE E AMPLITUDE NA FUNÇÃO “CONDUTIVIDADE ESPECTRAL" PARA ROCHAS, NO MODELO DE DIAS
}

\author{
Edivagner da Silva Ribeiro e Carlos Alberto Dias
}

Recebido em 29 julho, 2010 / Aceito em 23 novembro, 2011 Received on July 29, 2010 / Accepted on November 23, 2011

\begin{abstract}
This paper deals with the analysis of the relationship between the petrophysical coefficients and the shape of the curves of phase and amplitude of a "spectral conductivity" function (Dias' model) proposed to describe the electrical behaviour of rocks exhibiting the effect of induced electrical polarization. It starts with the development of the "total current conductivity" function set by this model and the petrophysical concepts attributed to its 5 parameters. The generated curves span all possibilities offered by the model and show that up to 2 peaks of phase and 3 plateaux in the amplitude of the conductivity can be provided. Such characteristic confers to the model a relatively great versatility for describing experimental data. The analysis of the possible combinations of the values of the 4 petrophysical parameters - with $\sigma_{0}$ being used only as a normalizing parameter for $\sigma$ - shows the influence of each parameter on the function and a correlation connecting them, evidencing the degree of the function sensitivity to its parameters' value intervals.
\end{abstract}

Keywords: petrophysics, induced polarization, electroconductivity, spectral conductivity, Dias' model.

RESUMO. Analisa-se, neste trabalho, a relação dos coeficientes petrofísicos com a forma das curvas de fase e amplitude da função "condutividade espectral" (modelo de Dias), descritiva do comportamento elétrico de rochas que apresentam o efeito de polarização elétrica induzida (efeito IP, do inglês electrical "induced polarization"). Inicia-se pelo desenvolvimento da função "condutividade de corrente total" do modelo e a conceituação do significado petrofísico de seus 5 parâmetros. As curvas geradas cobrem a totalidade das possibilidades oferecidas pelo modelo e mostram que podem existir até 2 picos na fase e até 3 patamares na amplitude da condutividade. Esta versatilidade confere ao modelo um maior potencial para descrever dados experimentais. A análise detalhada das possíveis combinações de valores entre os seus 4 parâmetros petrofísicos - sendo $\sigma_{0}$ usado apenas como parâmetro de normalização da função $\sigma$ - indica a influência de cada parâmetro na função e a existência de correlação entre eles, assim como, mostra o grau de sensibilidade da função associado aos diversos intervalos de valores dos seus parâmetros.

Palavras-chave: petrofísica, polarização induzida, eletrocondutividade, condutividade espectral, modelo de Dias. 


\section{INTRODUÇÃo}

Em um meio material, a condução da eletricidade é realizada por partículas carregadas, livres ou presas. As livres podem deslocar-se no interior do material sob a ação de um campo elétrico, sendo esse movimento contrariado pela força mecânica de "ação coletiva" do meio. As cargas presas (ou ditas "ligadas") têm movimento limitado a uma curta distância, e ao serem submetidas à influência de um campo elétrico tem sua distribuição espacial modificada. Tais partículas, sob a ação de um campo elétrico, sofrem um deslocamento de frações muito pequenas de um diâmetro molecular. Isso equivale a um deslocamento da carga "presa" positiva total em relação à carga negativa, de modo que 0 meio é dito estar polarizado (Panofsky \& Phillips, 2004).

Na escala atômico-molecular, em substâncias dielétricas constituídas por dipolos permanentes moleculares, um efeito de polarização é produzido pela orientação parcial desses dipolos (por ação de um campo elétrico) exclusivamente devido à movimentação de cargas "ligadas". Este tipo de polarização ficou bem caracterizado, com base em um modelo empírico, por uma função "constante dielétrica", dependente de frequência, proposta por Cole \& Cole (1941). Esta função é uma generalização do modelo original devido a Debye (Debye, 1929).

$\mathrm{Na}$ escala macroscópica, verifica-se em alguns materiais geológicos a presença de um tipo de polarização peculiar, denominada "polarização de interface", que ocorre na zona de contato metal-eletrólito ou argilomineral (ou zeólitos)-eletrólito. Este fenômeno é denominado efeito IP (electrical "induced polarization"), o qual vem sendo usado em exploração geofísica desde 1946 (Brant, 1959).

0 fenômeno IP já fora reconhecido pelos eletroquímicos, em laboratório, desde o final do século XIX, através da passagem de corrente elétrica num sistema composto metal-eletrólito, bem como a sua relação com 0 que no passado chamou-se de "sobrevoltagem" (overvoltage), um potencial-extra gerado pelo efeito IP. 0 gradiente deste potencial é proporcional à densidade de corrente por ele gerada, em consequência das barreiras eletroquímica e eletrocinética causadas na interface entre 0 metal ou argilomineral e a solução salina. Este potencial gerado pela polarização atua no sentido contrário ao do potencial ôhmico responsável pela acumulação de cargas inicial (Bockris \& Reddy, 1970).

0 termo "polarização induzida" tem por origem 0 fato de que este tipo de polarização não é permanente, só existindo enquanto o campo elétrico indutor estiver ligado, ou então, em forma transiente, imediatamente após cessar o campo indutor. Assim, significa em geofísica a polarização elétrica de interface produzida seja por uma corrente alternada, quando o fenômeno é observado no domínio da frequência, seja, correspondentemente, por um pulso elétrico, quando no domínio do tempo. Neste último caso, verificando-se uma voltagem-extra do tipo tempo-transiente (Madden \& Marshall, 1959a,b; Siegel, 1959; Wait, 1959a,b).

Na década de 60, houve uma grande expansão no uso deste método, em decorrência do seu enorme sucesso na exploração de depósitos de minerais metálicos disseminados e aquíferos subterrâneos, surgindo então diversos modelos para descrever esse fenômeno, na maioria empíricos. A primeira descrição analítica consistente do efeito de polarização elétrica induzida, contextualizada às equações de Maxwell do eletromagnetismo, foi proposta por Dias (1968; 1972), através de uma função complexa de frequência, condutividade de corrente total ou "condutividade espectral". Posteriormente, esta função foi reapresentada pelo mesmo autor sob nova reformulação (Dias, 2000). Este modelo partiu dos resultados parciais obtidos por eletroquímicos (Chang \& Jaffé, 1952; Grahame, 1952) e por geofísicos (Madden \& Marshall, 1959a,b; Wait, 1959a,b; Ward \& Fraser, 1967).

As possibilidades de aplicação da análise da "condutividade espectral", medida no laboratório ou no campo, ao estudo de temas de relevância na geofísica aplicada são várias, como por exemplo: distinção entre diferentes tipos e teores de minerais causadores da polarização, prospecção e caracterização de reservatórios de água subterrânea, distinção entre litologias constituintes do meio geológico (como arenito, arenito argiloso, foIhelho e rochas cristalinas), distinção entre zonas de formações geológicas preenchidas por fluidos diversos (como água, óleo ou gás), ou, ainda, variações do espaço intergranular das fontes geradoras do efeito IP no interior da matriz da rocha (Pelton et al., 1978; Olhoeft, 1985; Mahan et al., 1986; Lima, 1995; Dias, 2000; Lima et al., 2005; Dias et al., 2005, 2006). Outras aplicações, mais recentes, ainda não totalmente consolidadas, são a determinação in situ da permeabilidade e a distribuição espacial da dimensão dos poros da rocha em volume (Lima, 1995; Lima \& Niwas, 2000; Maosong et al., 2004, 2006a,b; Maosong \& Honggen, 2008).

Dada a relevância do modelo de Dias, dentre os modelos vigentes mais usados para descrição do efeito IP, no contexto da E\&P de hidrocarbonetos e, sobretudo, na modelagem e inversão numérica de medidas eletromagnéticas (Morrison et al., 1969; Cockburn, 1983; Dias et al., 2005, 2006; Fainberg et al., 2009; Yue et al., 2009), o presente artigo se propõe analisar o comportamento da função "condutividade espectral" desse modelo. 
(a)

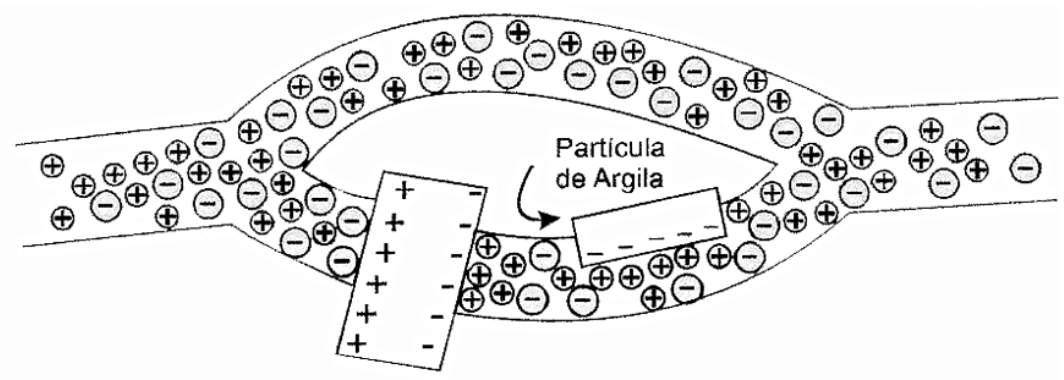

Partícula metálica

$R$

(b)

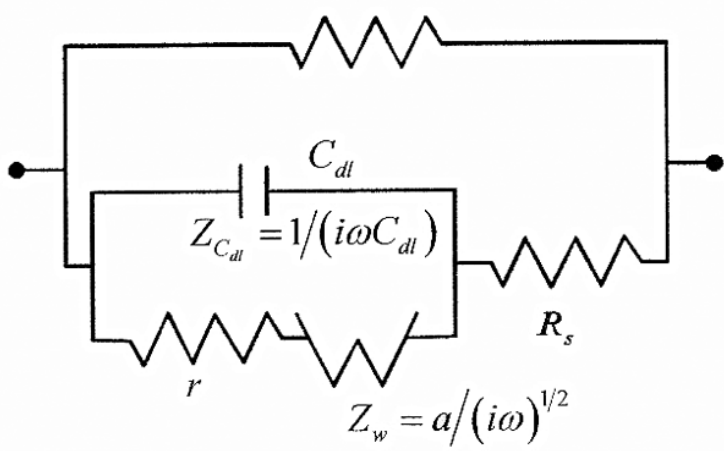

Figura 1 - (a) Esquema de rocha eletricamente polarizada (IP) a baixa frequência (Dias, 2000); (b) Circuito fundamental da "célula unitária" do comportamento elétrico de uma rocha eletricamente polarizada (Dias, 1968, 1972, 2000).

Esta análise é feita através da representação gráfica das funções típicas de fase e amplitude da condutividade. Procurando preencher uma lacuna, a presente análise procura enriquecer a percepção intuitiva sobre o comportamento dessa função, quando os seus parâmetros petrofísicos assumem valores cobrindo os seus intervalos característicos e a frequência varia em todo 0 espectro em que ocorre o fenômeno IP $\left(10^{-4} \mathrm{~Hz}\right.$ a $\left.1 \mathrm{MHz}\right)$.

\section{O MODELO DE DIAS: FUNÇÃO “CONDUTIVIDADE ESPECTRAL”, OU DE CORRENTE TOTAL}

Dias (1968) verificou que 0 circuito analógico proposto por Grahame (1952), para descrever o comportamento elétrico da zona de contato de uma única placa metálica com uma solução salina, ao ser associado em paralelo com um resistor, gerava um "circuito elementar" do qual deriva uma função condutividade complexa capaz de descrever o parâmetro de proporcionalidade entre a densidade de corrente total e o campo elétrico, em dados experimentais em testemunhos de rocha, quando ocorre polarização. Esse fato o levou a admitir que este tipo de polarização ocorre nas rochas, tendo esse "circuito elementar" como uma espécie de "célula unitária" do comportamento elétrico do material, repetindo-se indefinidamente no volume da rocha.
A Figura 1 mostra a representação de uma "célula unitária" do comportamento elétrico de uma rocha eletricamente polarizada, onde a presença de partículas de argila ou metálicas disseminadas, em contato com a solução salina, gera surgimento de uma estrutura elétrica conhecida como dupla camada elétrica de Helmholtz, resultando na formação de acúmulos de carga na vizinhança de cada superfície de contato, responsáveis por efeitos capacitivos e de difusão iônica, competindo com a condução puramente ôhmica. 0 circuito analógico descrito na Figura 1(b) passou a ser chamado de "circuito fundamental", ou "célula elementar do comportamento elétrico" do meio polarizável (Dias, 2000).

Tem-se na Figura 1:

$Z^{\prime}$ : parte integrante do membro inferior do circuito da Figura $1(b)$, representativo do circuito equivalente à zona de contato entre uma partícula metálica e o eletrólito. Segundo Grahame (1952), é uma associação de um efeito capacitivo da dupla camada elétrica $\left(Z_{C_{d l}}\right)$ em paralelo com uma composição em série de uma resistência $r$, descritiva de condução ôhmica, com uma impedância característica $\left(Z_{w}\right)$, descritiva de condução por difusão de íons, no interior da dupla camada elétrica; 
$R_{S}$ : resistor em série com $Z^{\prime}$, indicativo de um segmento constituído apenas por eletrólito, livre da influência da perturbação gerada pela dupla camada elétrica;

$R$ : resistor em paralelo com $R_{S}+Z^{\prime}$, indicativo da possibilidade de passagem de corrente por um caminho ôhmico alternativo (livre de polarização). Este resistor foi acrescentado por Dias $(1968,1972)$ ao "circuito elementar" de uma zona de contato, tornando-se elemento obrigatório no contexto de um meio poroso.

Com base nesse circuito, escreve-se a função admitância

$$
\frac{1}{Z}=\frac{1}{R}+\frac{1}{R_{S}+Z^{\prime}}
$$

onde $Z^{\prime}$ pode ser escrito como

$$
\frac{1}{Z^{\prime}}=\frac{1}{Z_{C_{d l}}}+\frac{1}{r+Z_{w}},
$$

em que $Z_{C_{d l}}$ representa a impedância capacitiva da dupla camada elétrica de Helmholtz e $Z_{w}$ a impedância de Warburg, esta última associada ao processo de difusão (Bockris \& Reddy, 1970). Estas impedâncias vêm dadas, respectivamente, por $1 /\left(i \omega C_{d l}\right)$ e $a /(i \omega)^{1 / 2}$, sendo $a$ um parâmetro característico.

Então, a função "condutividade espectral" normalizada pelo seu valor $d c$, representado por $\sigma_{0}$, é dada por

$$
\frac{\sigma}{\sigma_{0}}=1+\frac{R}{R_{s}+\frac{\left(r+Z_{w}\right) Z_{C_{d l}}}{r+Z_{w}+Z_{C_{d l}}}}
$$

que, após certo trabalho algébrico (ver Apêndice), reduz-se a:

$$
\frac{\sigma}{\sigma_{0}}=1+\frac{R \lambda / Z_{w}}{1+\left(r+R_{S}\right) \lambda^{\prime} / Z_{w}}
$$

onde

$$
\begin{aligned}
\lambda & =1+\mu, \\
\lambda^{\prime} & =1+\left(1-\frac{r}{r+R_{S}}\right) \mu, \\
\mu & =\frac{r+Z_{w}}{Z_{C_{d l}}}
\end{aligned}
$$

e

$$
1 / Z=g \sigma \quad \text { e } \quad 1 / R=g \sigma_{0}
$$

com $g=S / d$, sendo $S$ a seção reta de um testemunho de rocha e $d$ o seu comprimento.

Dias $(1968,1972)$ escreveu a sua primeira versão da função (4) usando cinco parâmetros, alguns dos quais com intervalos de variação não-definidos, podendo por isso inclusive gerar valores espúrios para a condutividade. Posteriormente, Dias (2000) reescreveu essa função em forma definitiva, usando somente parâmetros com intervalos de variação bem definidos, garantindo a sua unicidade, e com significado petrofísico assegurado.

Os novos parâmetros introduzidos são:

- $\sigma_{0}$ (condutividade $d c$ ): corresponde ao valor mais baixo da condutividade, quando o efeito da polarização atinge sua máxima saturação;

- $m$ (cargabilidade): medida da máxima dispersão da condutividade, normalizada pelo maior valor do módulo de $\sigma$. Este máximo valor de $\sigma$ ocorre às altas frequências, às quais 0 efeito IP ainda não se faz presente. A cargabilidade corresponde à medida da intensidade do efeito da polarização existente, definida como (Siegel, 1959; Wait, 1959b)

$$
m=\frac{\sigma_{\omega \rightarrow \infty}-\sigma_{\omega \rightarrow 0}}{\sigma_{\omega \rightarrow \infty}}
$$

No circuito da célula elementar, a cargabilidade corresponde a $m=R /\left(R+R_{S}\right)$. Na escala macroscópica, vem relacionada com o tipo/espécie da fonte geradora do efeito e, através de uma função peculiar, ao seu teor na rocha (Pelton et al., 1978). Trata-se do parâmetro melhor estabelecido na literatura, na interpretação do efeito IP. Este parâmetro é adimensional e está definido no intervalo $0 \leq m<1$.

- $\tau$ (tempo de relaxação): corresponde ao tempo de relaxação da zona da dupla camada elétrica de Helmholtz, relacionado com o decaimento temporal do efeito quando a difusão é suplantada pelos outros mecanismos de condução na célula elementar, sendo dado por

$$
\tau=r C_{d l} .
$$

Trata-se de um parâmetro com dimensão de tempo, normalmente menor que $1 \mathrm{~s}$, podendo baixar a até $10^{-7} \mathrm{~S}$. $\mathrm{Na}$ escala macroscópica, vem relacionado com o tamanho do grão mineral da fonte geradora do efeito (Olhoeft, 1985). É o segundo melhor estabelecido parâmetro na literatura, na interpretação do efeito IP;

- $\delta$ (fração do comprimento da célula elementar afetada pelo efeito de polarização), dada por

$$
\delta=\frac{r}{r+R_{s}} .
$$

Este parâmetro é também adimensional e definido no intervalo $0 \leq \delta<1$, sendo peculiar deste modelo. Envolve 
apenas os componentes ôhmicos da condução na célula elementar, podendo $r$ ser relacionado aproximadamente ao comprimento da zona afetada pela polarização e $R_{S}$ ao comprimento da porção da célula livre dessa influência. No contexto de uma rocha, $(1-\delta)$ pode ser associado ao espaçamento entre duas partículas vizinhas geradoras do efeito IP.

- $\eta$ (parâmetro eletroquímico), dado por

$$
\eta=\frac{a}{r}
$$

Este parâmetro é também peculiar deste modelo. Tem a dimensão de $s^{-1 / 2}$ e acha-se relacionado à significância relativa entre a componente ôhmica da corrente elétrica e o módulo da componente da corrente produzida por difusão na frequência de $1 \mathrm{rad} / \mathrm{s}$, dentro da espessura da dupla camada elétrica, na célula elementar. Possui, portanto, uma relação direta com o processo cinético produtor da polarização. Na célula elementar, pode ser igualado a $2 D^{1 / 2} / \ell$ onde $D$ é 0 valor da difusividade do cátion da solução e $\ell$ é a espessura da dupla-camada elétrica de Helmholtz (Chang \& Jaffé, 1952).

Sendo $\eta$ dependente de $\ell$, não variará com a frequência $\omega$ só em primeira aproximação, em decorrência da linearização do mecanismo da condução. Dias (2000) sugere que, para as rochas, $\eta$ pode variar desde valores da ordem de $0,1 s^{-1 / 2}$ até aproximadamente $150 \mathrm{~s}^{-1 / 2}$. E esperase que $\eta$ cresça com a concentração tanto das fontes disseminadas quanto dos íons em solução, em menor intensidade com 0 último.

Introduzindo os novos parâmetros, pode-se escrever após certo exercício algébrico,

$$
\begin{aligned}
\lambda & =1+\mu, \\
\lambda^{\prime} & =1+(1-\delta) \mu, \\
\mu & =i \omega \tau\left[1+\eta(i \omega)^{-1 / 2}\right],
\end{aligned}
$$

resultando em

$$
\begin{aligned}
& \frac{R}{Z_{w}}=\left(m \frac{(1-\delta)}{(1-m)}\right)(\eta \delta)^{-1}(i \omega)^{1 / 2}, \\
& \frac{r+R_{s}}{Z_{w}}=(\eta \delta)^{-1}(i \omega)^{1 / 2},
\end{aligned}
$$

podendo-se, finalmente, escrever

$$
\sigma=\sigma_{0}\left[1+\alpha \frac{\lambda \beta(i \omega)^{1 / 2}}{1+\lambda^{\prime} \beta(i \omega)^{1 / 2}}\right]
$$

onde $\lambda$, $\lambda^{\prime}$ e $\mu$ são dados por (13), (14) e (15), respectivamente, $\mathrm{e}$

$$
\begin{aligned}
& \alpha=m(1-\delta) /(1-m), \\
& \beta=(\eta \delta)^{-1} .
\end{aligned}
$$

De acordo com Dias (2000), existem na literatura 12 modelos principais propostos para descrever 0 comportamento elétrico de um testemunho de rocha, em laboratório, relacionado ao efeito IP. São modelos que oferecem, com maior ou menor grau de consistência e confiabilidade, parâmetros petrofísicos para interpretação de características e propriedades do meio. Demonstra 0 autor nesse trabalho que seu modelo é provavelmente 0 mais apropriado para descrever 0 efeito IP, por possuir três características básicas:

a) ser capaz e versátil, dentre os melhores, para superpor dados experimentais, com a vantagem de fazê-lo usando o menor número de coeficientes;

b) ser fenomenológico de origem, isto é, construído a partir dos princípios fundamentais que regulam o fenômeno e 0 comportamento do meio que exibe 0 efeito IP sob a ação de um campo elétrico harmônico aplicado;

c) ser expresso através de coeficientes que possuem unicidade de valores, definidos em intervalos pré-estabelecidos de variação, e significado petrofísico consistente.

Tem-se, portanto, uma função complexa $\sigma$, dependente de cinco parâmetros $\left(\sigma_{0}, \tau, \eta, \delta, m\right)$ e da variável frequência $\omega(\omega=2 \pi f)$.

0 modelo ora apresentado tem sido objeto de análises (Cockburn, 1983; Bamberg et al., 1989) e usado em modelagem visando a detecção eletromagnética do efeito IP (Morrison et al., 1969; Xusan et al., 2001; Hashsish, 2002; Zhu et al., 2005; Fainberg et al., 2009; Yue et al., 2009). Não obstante, existe uma lacuna na compreensão intuitiva do comportamento desta função, que se procura cobrir no presente trabalho.

\section{METODOLOGIA}

Os gráficos que serão apresentados, para fase e amplitude da função "condutividade de corrente total" (modelo de Dias) versus frequência, foram construídos usando 100 valores de frequência, espaçados com regularidade em escala logarítmica, no intervalo de $10^{-4} \mathrm{~Hz}$ até $1 \mathrm{MHz}$.

Com 0 objetivo de mostrar a correlação entre os valores dos parâmetros e a desenvoltura dos tipos de curvas geradas a 


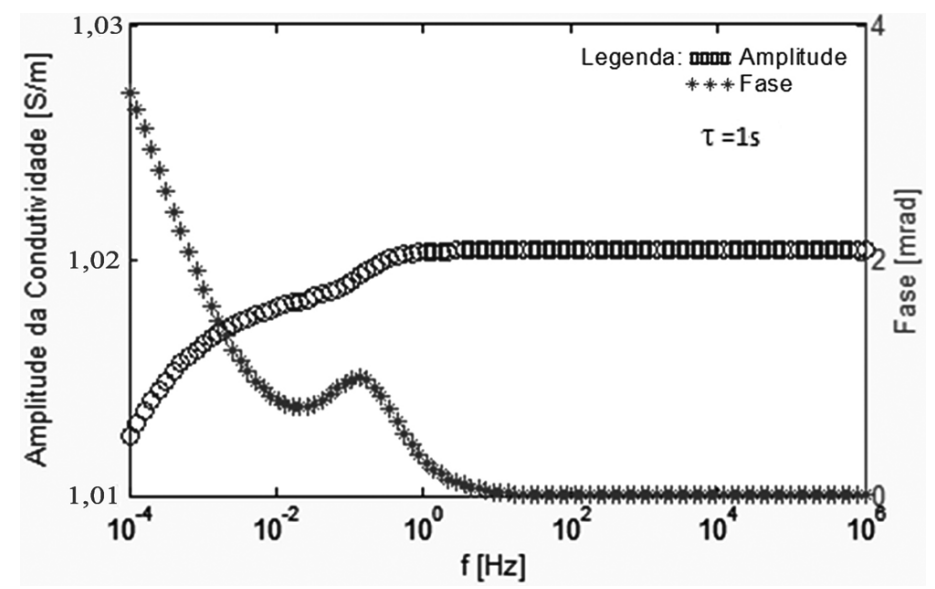

Figura 2 - Curvas de amplitude e fase da função condutividade de corrente total normalizada, $\operatorname{com}\left(\eta=0,15 \mathrm{~s}^{-1 / 2} ; \delta=0,09 ; m=0,02\right)$ e $\tau=1 \mathrm{~s}$.

Tabela 1 - Distribuição dos valores dos parâmetros mantidos fixos ou variáveis, nos seus intervalos de variação, usados na geração de curvas de fase e amplitude.

\begin{tabular}{|r|c|c|c|c|c|}
\hline $\begin{array}{c}\text { Grupo e } \\
\text { subgrupo }\end{array}$ & $\begin{array}{c}\text { Valor fixo } \\
\text { baixo }\end{array}$ & $\begin{array}{c}\text { Valor fixo } \\
\text { intermediário }\end{array}$ & $\begin{array}{c}\text { Valor fixo } \\
\text { elevado }\end{array}$ & $\begin{array}{c}\text { Parâmetro } \\
\text { variável }\end{array}$ & Figura \\
\hline $1^{\circ} \mathrm{G}-1 . \mathrm{a}$ & $(\delta, m) \eta$ & - & - & $\tau$ & 3 \\
$-1 . \mathrm{b}$ & $(\delta, m)$ & - & $\tau$ & $\eta$ & 4 \\
$-1 . \mathrm{c}$ & $(\delta, m) \tau$ & - & - & $\eta$ & 5 \\
$-1 . \mathrm{d}$ & $(\delta, m)$ & $\tau$ & - & $\eta$ & 6 \\
$2^{\circ} \mathrm{G}$ & $(\tau, m, \eta)$ & - & - & $\delta$ & 7 \\
$3^{\circ} \mathrm{G}$ & $(\tau, m)$ & - & $\delta$ & $\eta$ & 8 \\
$4^{\circ} \mathrm{G}$ & - & $(\tau, \eta, \delta)$ & - & $m$ & 9 \\
\hline
\end{tabular}

partir da função condutividade, manteve-se fixo 0 intervalo de frequências, deixando-se evoluir as curvas de fase e amplitude e variar os valores dos parâmetros. 0 valor de $\sigma_{0}$ foi mantido constante e igual a $1 \mathrm{~S} / \mathrm{m}$, e os demais parâmetros $(\tau, \eta, \delta, m)$ variando, um por vez, em seus intervalos próprios.

Visando cobrir a totalidade dos casos, evitando proliferação de curvas desnecessariamente, partiu-se de um caso ilustrativo que mostra as características e tendências gerais das funções analisadas, daí progredindo para as curvas específicas. 0 caso genérico vem apresentado na Figura 2, com valores fixos baixos para os parâmetros $(\delta, m, \eta)$, respectivamente $(0,09 ; 0,02$; $\left.0,15 s^{-1 / 2}\right)$, e $\tau$ elevado (1s).

Os demais casos seguiram uma distribuição conveniente dos valores dos parâmetros nos seus intervalos de variação, arrumados em quatro grupos, mostrados na Tabela 1.

Foram evitados, por não acrescentar informação nova, os gráficos gerados com valores fixos baixos de $(\delta, \tau)$ e elevado de $m$, deixando $\eta$ como parâmetro variável, em relação ao caso em que $m$ é um valor fixo baixo (subgrupo 1.c). Bem como, os gráficos gerados com valores fixos elevados de $(\tau, \eta, \delta)$, deixando $m$ como parâmetro variável, em relação ao caso do $4^{\circ} \mathrm{G}$.

Após construção desses conjuntos de gráficos, analisa-se 0 comportamento das curvas de fase, tomando por base a formação dos máximos e a sua posição no espectro de frequências e as curvas de amplitude com base na formação de patamares e no próprio formato da curva.

\section{RESULTADOS E DISCUSSÕES}

0 modelo proposto por Dias (2000) é uma função de frequência que possui cinco parâmetros, constantes para cada amostra de rocha. 0 valor de $\sigma_{0}$ é a leitura do valor assintótico mínimo da amplitude da condutividade versus frequência. Os demais parâmetros possuem valores situados nos intervalos específicos, descritos anteriormente. 0 efeito da variação de cada um dos 4 parâmetros $(\tau, \eta, \delta, m)$ será analisado a seguir, mostrando a influência de cada um deles nas curvas da função. 

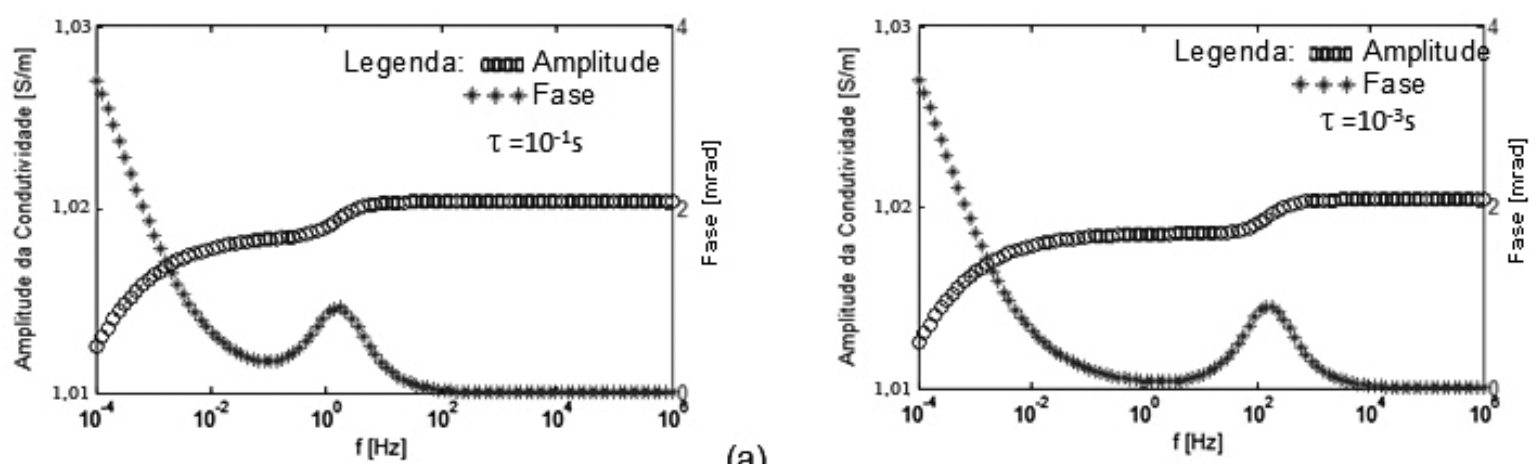

(a)

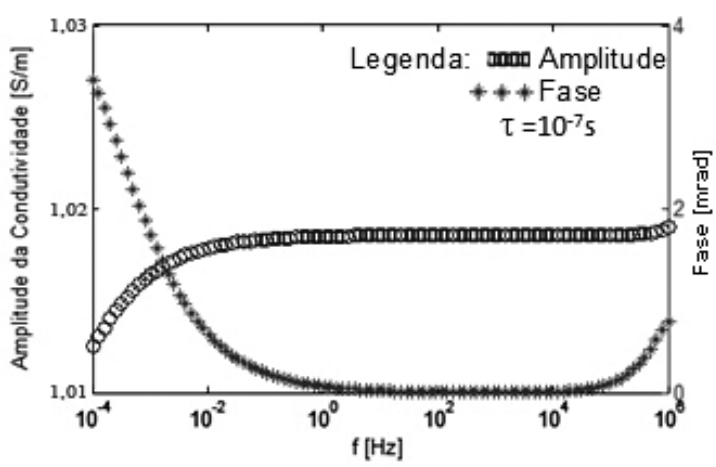

(b)

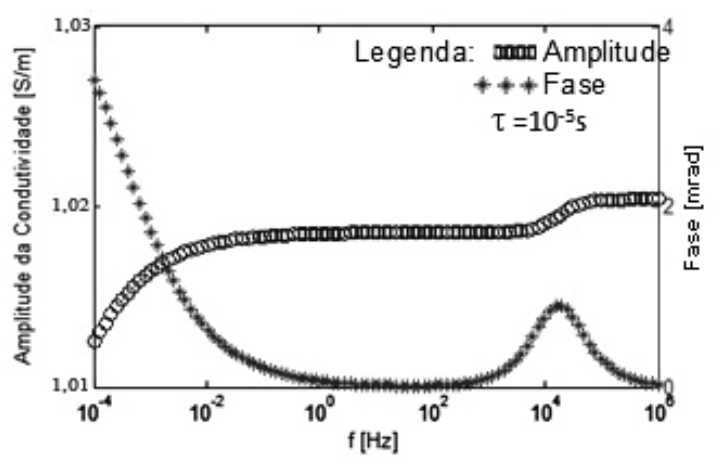

(c)

$f[\mathrm{~Hz}]$

Figura 3 - Curvas de amplitude e fase da função condutividade de corrente total normalizada, com variação de $\tau$ : (a) $\tau=10^{-1} \mathrm{~s}, \eta=0,15 \mathrm{~s}^{-1 / 2}, \delta=0,09$ e $m=0,02$; (b) $\tau=10^{-3} \mathrm{~s}, \eta=0,15 \mathrm{~s}^{-1 / 2}, \delta=0,09$ e $m=0,02$; (c) $\tau=10^{-5} \mathrm{~s}, \eta=0,15 \mathrm{~s}^{-1 / 2}, \delta=0,09$ e $m=0,02$; (d) $\tau=10^{-7} \mathrm{~s}, \eta=0,15 \mathrm{~s}^{-1 / 2}$, $\delta=0,09$ e $m=0,02$.

As curvas típicas da função condutividade terão variação restrita ao intervalo de frequências $10^{-4} \mathrm{~Hz}$ a $1 \mathrm{MHz}$. Fora deste intervalo, a discussão é descartada pelo fato de aí não existir 0 fenômeno IP, conforme Dias (2000).

Mantendo-se $(\eta, \delta, m)$ pequenos, respectivamente $\left(0,15 \mathrm{~s}^{-1 / 2} ; 0,09 ; 0,02\right)$, com $\tau$ no limite superior (1s), podese observar na Figura 2 a existência do primeiro pico de fase à baixa frequência (visto que para frequência aproximando-se de zero a fase deverá convergir para zero) e o segundo pico situado à frequência de $10^{-1} \mathrm{~Hz}$. Conforme será visto, na totalidade das curvas das figuras mostradas, a fase neste modelo pode apresentar até dois máximos.

Diminuindo $\tau$, mantendo fixos e pequenos os valores dos demais parâmetros, o segundo pico da fase desloca-se para as frequências maiores, conservando sua intensidade (Fig. 3), iniciando um pouco acima de $1 \mathrm{~Hz}$ quando $\tau=10^{-1} \mathrm{~s}$ e de $1 \mathrm{MHz}$ quando $\tau=10^{-7} \mathrm{~s}$. Pode-se inferir, então, uma generalização na associação da posição do segundo pico em $10^{n-1} \mathrm{~Hz}$ com 0 valor do tempo de relaxação em $\tau=10^{-n} \mathrm{~s}$. Portanto, a influência do parâmetro $\tau$, nessa configuração dos valores dos parâmetros, é responsável apenas pelo deslocamento da frequência em que ocorre 0 segundo pico.

Por outro lado, a Figura 4 mostra que 0 parâmetro eletroquímico $(\eta)$ influencia no posicionamento do primeiro pico de fase. 0 deslocamento deste pico fica limitado pela posição do segundo pico de fase, que por sua vez tem sua posição controlada pelo valor de $\tau$, conforme visto na Figura 3. Para determinados intervalos de valores dos parâmetros $\tau$ e $\eta$, o primeiro $\mathrm{e}$ 0 segundo picos de fase se sobrepõem (Fig. 4, (c) em diante), de modo que a intensidade desse pico único (soma dos dois picos) passa a ser maior, tornando difícil identificar a contribuição individual de cada um desses parâmetros na formação da curva. Para $\tau$ grande $(\tau=0,5 \mathrm{~s})$, aumentando $\eta$ de $0,3 \mathrm{~s}^{-1 / 2}$ a $150 \mathrm{~s}^{-1 / 2}$, o primeiro pico se desloca até aproximadamente $10^{-1} \mathrm{~Hz}$, onde ocorre a soma das intensidades dos picos, passando a diminuir sua largura e aumentando a intensidade do pico de fase (Fig. 4).

De acordo com a Figura 5, quando $\tau$ é pequeno $\left(\tau=10^{-6} \mathrm{~s}\right)$, deixando-se variar $\eta$ desde $1 \mathrm{~s}^{-1 / 2}$ até $150 \mathrm{~s}^{-1 / 2}, 0$ segundo pico fica fixo em torno da frequência de $10^{5} \mathrm{~Hz}$ e com a mesma inten- 

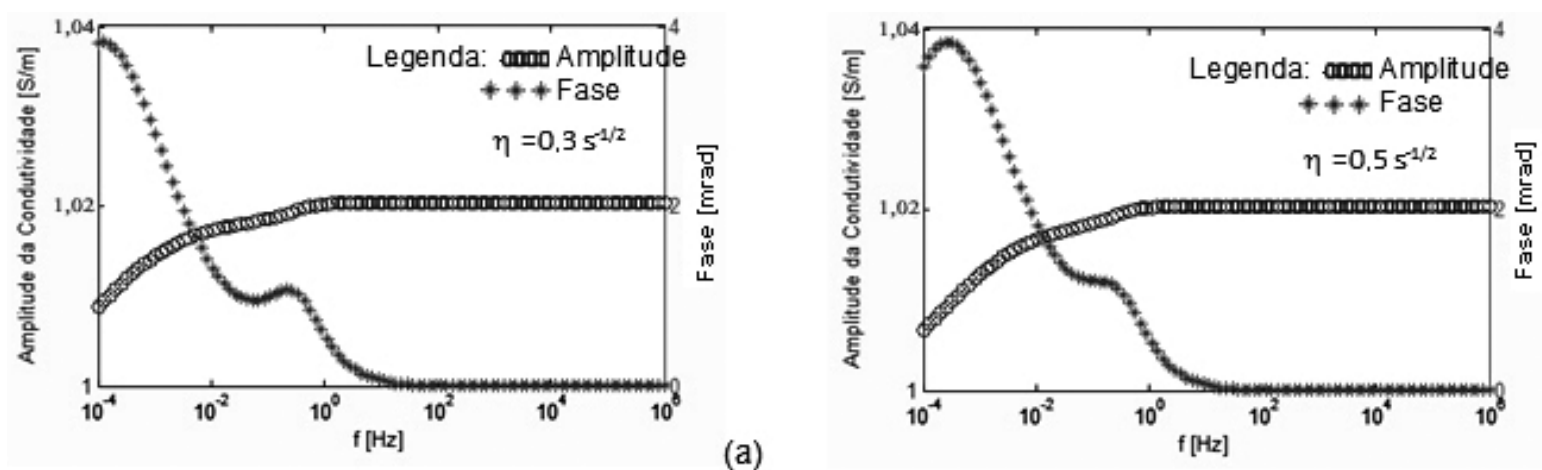

(b)
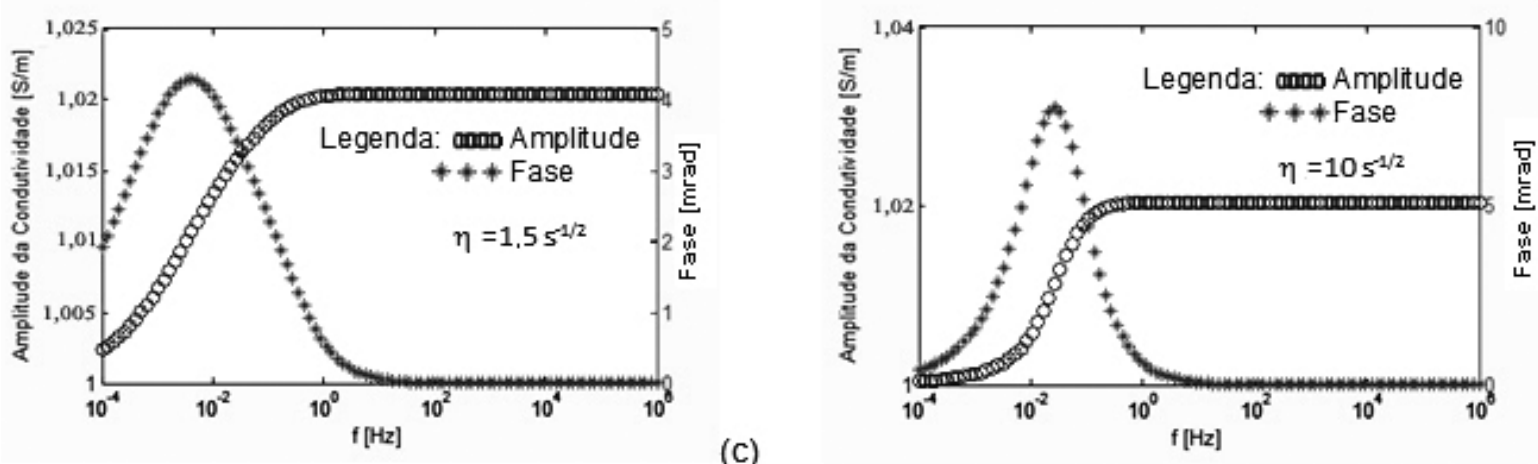

(d)
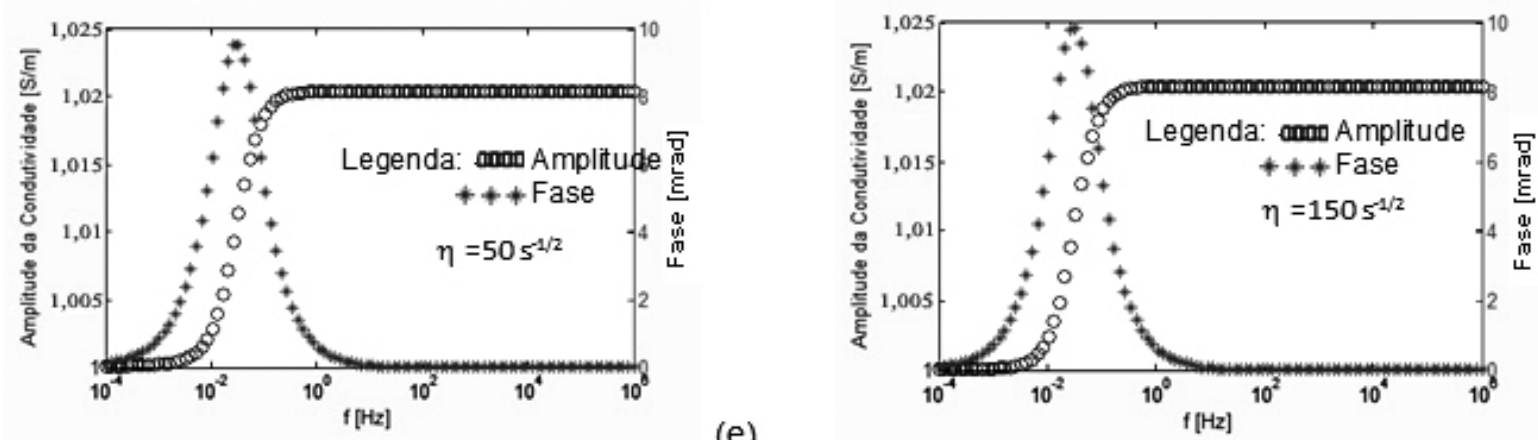

(f)

Figura 4 - Curvas de amplitude e fase da função condutividade de corrente total normalizada, com variação de $\eta \operatorname{com} \tau=0,5 \mathrm{~s}$ : (a) $\tau=0,5 \mathrm{~s}, \eta=0,3 \mathrm{~s}^{-1 / 2}$, $\delta=0,09$ e $m=0,02$; (b) $\tau=0,5 \mathrm{~s}, \eta=0,5 \mathrm{~s}^{-1 / 2}, \delta=0,09$ e $m=0,02$; (c) $\tau=0,5 \mathrm{~s}, \eta=1,5 \mathrm{~s}^{-1 / 2}, \delta=0,09$ e $m=0,02$; (d) $\tau=0,5 \mathrm{~s}, \eta=10 \mathrm{~s}^{-1 / 2}$, $\delta=0,09$ e $m=0,02$; (e) $\tau=0,5 \mathrm{~s}, \eta=50 \mathrm{~s}^{-1 / 2}, \delta=0,09$ e $m=0,02$; (f) $\tau=0,5 \mathrm{~s}, \eta=150 \mathrm{~s}^{-1 / 2}, \delta=0,09$ e $m=0,02$.

sidade. Já o primeiro pico desloca-se de $10^{-3} \mathrm{~Hz}$ até a frequência de $10^{2} \mathrm{~Hz}$. Se $\delta$ e $m$ são pequenos ( $\left.\ll 1\right)$, não ocorre soma das intensidades dos picos de fase (Fig. 5). Verifica-se na Figura 5 que 0 deslocamento do primeiro pico da fase para a direita se mostra muito sensível a pequenas variações de $\eta$, entre valores pouco maiores que $1 \mathrm{~s}^{-1 / 2}$ até aproximadamente $30 \mathrm{~s}^{-1 / 2}$. Os valores de $\eta$ entre $30 \mathrm{~s}^{-1 / 2}$ e $150 \mathrm{~s}^{-1 / 2}$ contribuem mais suavemente para 0 deslocamento do primeiro pico (Figs. 5(c), (d), (e) e (f)). Conclui-se que a posição do primeiro pico de fase é controlada por $\eta$, enquanto a posição do segundo pico é controlada por $\tau$, com a posição do primeiro pico não fazendo ultrapassagem em relação ao segundo pico.

De acordo com a Figura 6, verifica-se que para valores intermediários do tempo de relaxação, como $\tau=10^{-3} \mathrm{~s}$, tem-se a formação do segundo pico de fase próximo à frequência de $10^{2} \mathrm{~Hz}$. 0 primeiro pico, na Figura 6 , inicia-se em $10^{-3} \mathrm{~Hz}$ para $\eta=1 \mathrm{~s}^{-1 / 2}$, deslocando-se para $10^{-1} \mathrm{~Hz} \operatorname{com} \eta=10 \mathrm{~s}^{-1 / 2}$, ocorrendo a partir de $\eta=30 \mathrm{~s}^{-1 / 2}$ a composição dos dois picos de fase, preservando-se 0 valor da fase herdado do segundo pico em $10^{2} \mathrm{~Hz}$. 


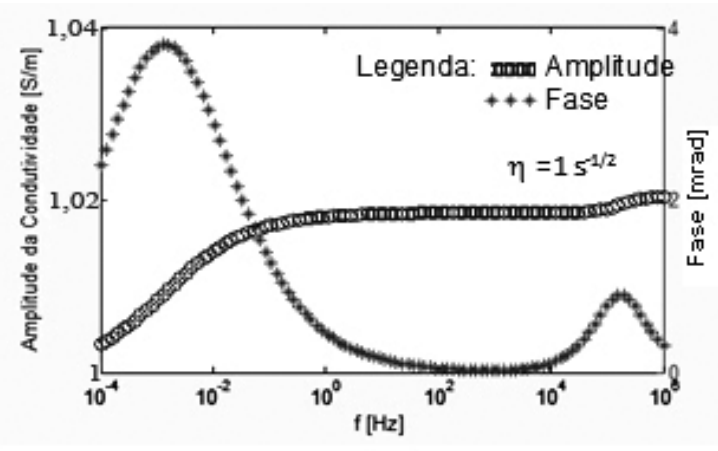

(a)

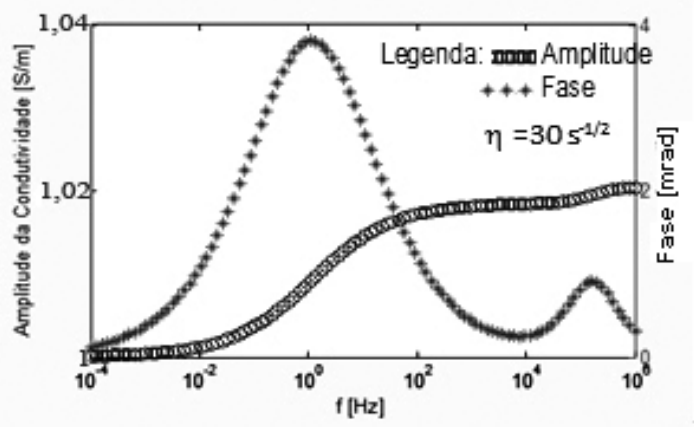

(c)

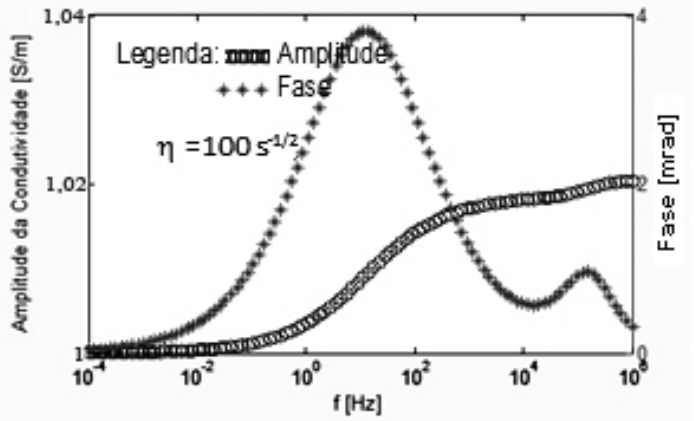

(e)

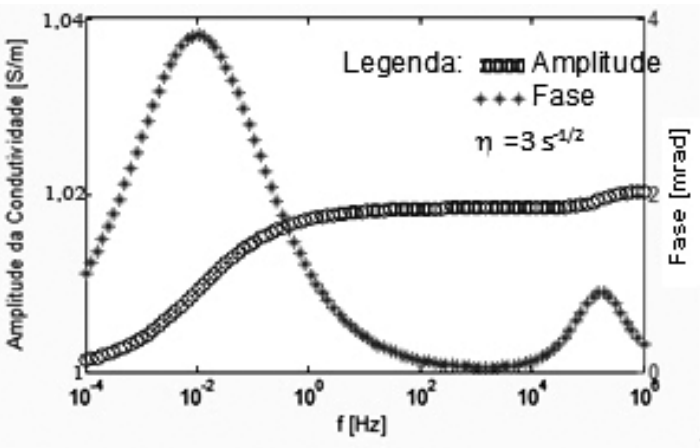

(b)

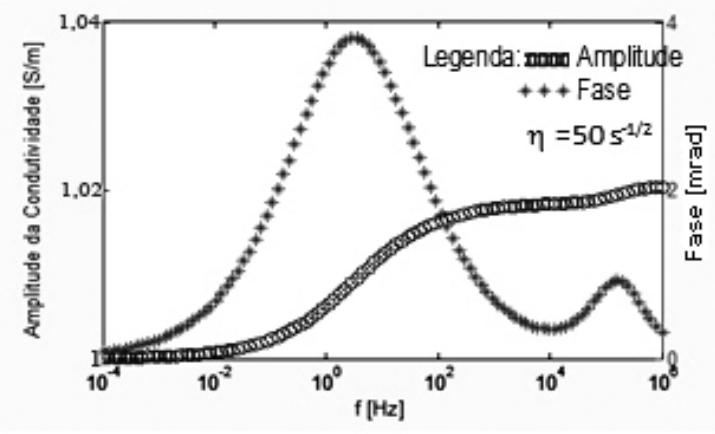

(d)

Figura 5 - Curvas de amplitude e fase da função condutividade de corrente total normalizada, com variação de $\eta$ com $\tau=1 \times 10^{-6} \mathrm{~S}$ : (a) $\tau=1 \times 10^{-6} \mathrm{~S}$, $\eta=1 \mathrm{~s}^{-1 / 2}, \delta=0,09$ e $m=0,02$; (b) $\tau=1 \times 10^{-6} \mathrm{~s}, \eta=3 \mathrm{~s}^{-1 / 2}, \delta=0,09$ e $m=0,02$; (c) $\tau=1 \times 10^{-6} \mathrm{~s}, \eta=30 \mathrm{~s}^{-1 / 2}, \delta=0,09$ e $m=0,02$; (d) $\tau=1 \times 10^{-6} \mathrm{~S}, \eta=50 \mathrm{~s}^{-1 / 2}, \delta=0,09$ e $m=0,02$; (e) $\tau=1 \times 10^{-6} \mathrm{~S}, \eta=100 \mathrm{~s}^{-1 / 2}, \delta=0,09$ e $m=0,02$; (f) $\tau=1 \times 10^{-6} \mathrm{~S}, \eta=150 \mathrm{~s}^{-1 / 2}, \delta=0,09$ e $m=0,02$.

Verifica-se, na Figura 7, que o parâmetro $\delta$ promove uma compensação nos valores do primeiro e do segundo picos de fase, isto é, o segundo pico aumenta enquanto o primeiro diminui, quando temos fixos $m$ e $\tau$ pequenos. Para $\delta$ pequeno, como 0,09, (Fig. 6(a)), o primeiro pico de fase apresenta-se às baixas frequências $\left(10^{-2} \mathrm{~Hz}\right)$ com sua intensidade máxima e 0 segundo pico, de menor intensidade, vem posicionado à alta frequência $\left(10^{5} \mathrm{~Hz}\right)$. Aumentando 0 valor de $\delta, 0$ primeiro pico desloca-se para a direita, diminuindo a intensidade, atingindo valores próximos de zero com $\delta$ da ordem de 0,95, enquanto 0 segundo pico cresce na intensidade e desloca-se para a direita, de $10^{5} \mathrm{~Hz}$ até $10^{6} \mathrm{~Hz}$, atingindo nesta frequência 0 seu valor máximo, como apresentado na Figura 7(d). A amplitude da condutividade mostra três patamares para $\delta$ variando de 0,09 a 0,3 . Para valores de $\delta$ acima de 0,6 , verificam-se dois patamares, seguidos de um ramo ascendente da curva entre $10^{5} \mathrm{~Hz}$ e $10^{6} \mathrm{~Hz}$. 

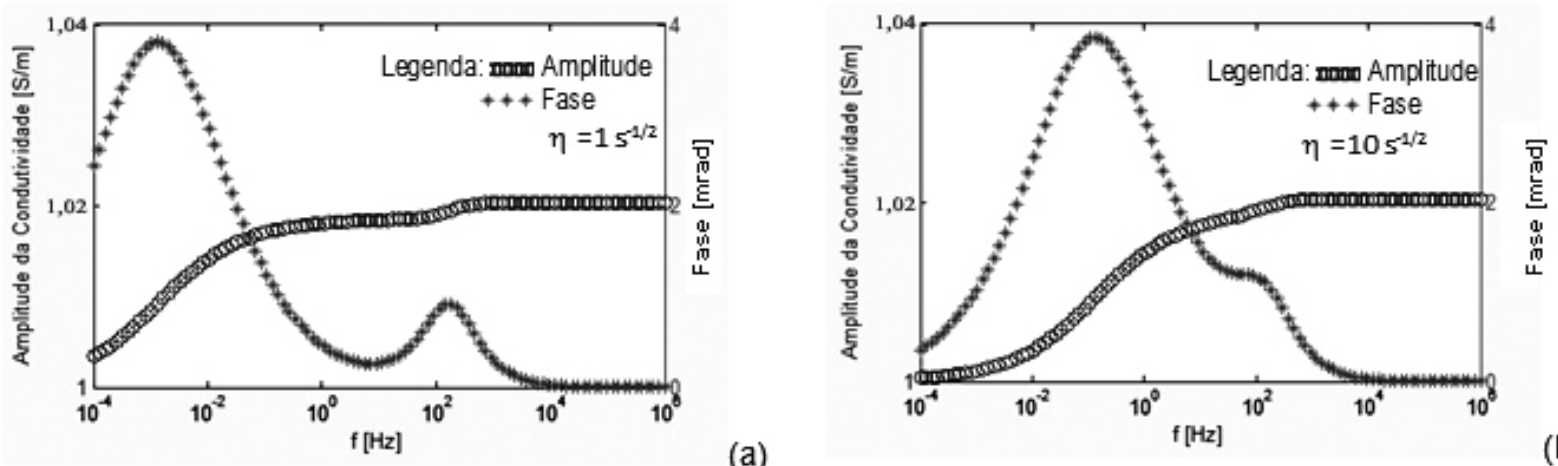

(b)
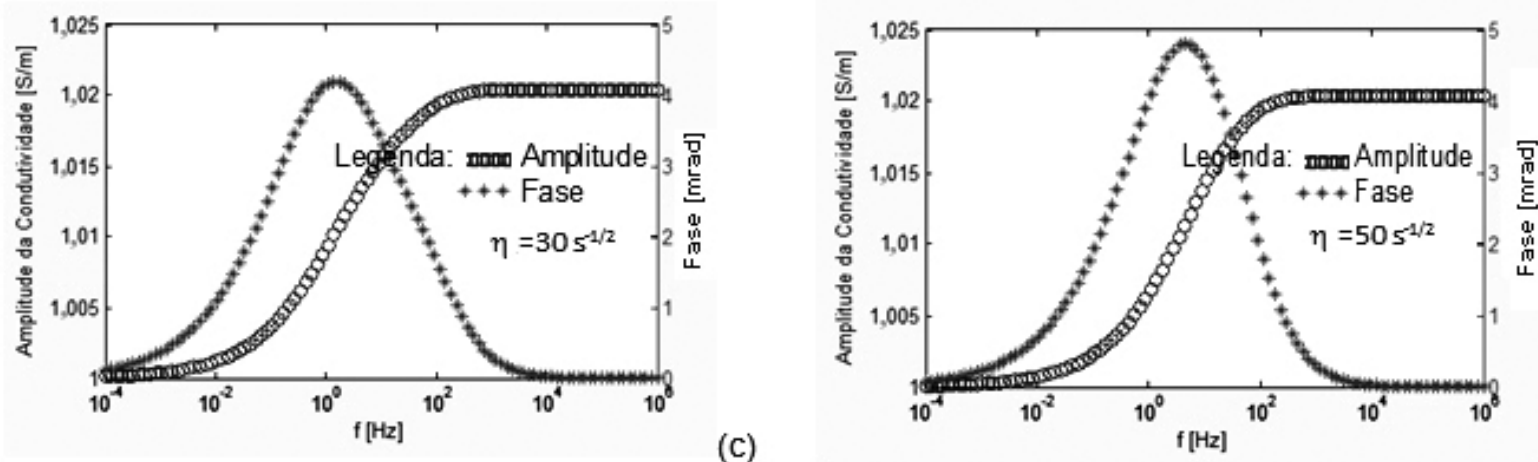

(d)
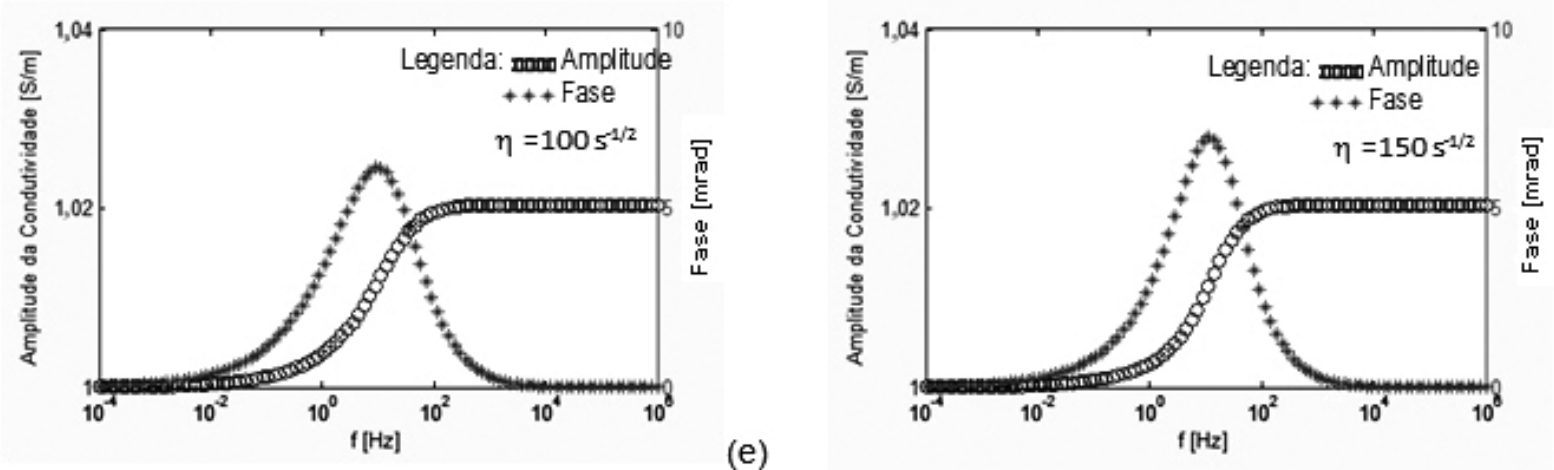

(f)

Figura 6 - Curvas de amplitude e fase da função condutividade de corrente total normalizada, com variação de $\eta$ com $\tau=1 \times 10^{-3} \mathrm{~s}$ : (a) $\tau=1 \times 10^{-3} \mathrm{~s}$, $\eta=1 \mathrm{~s}^{-1 / 2}, \delta=0,09$ e $m=0,02$; (b) $\tau=1 \times 10^{-3} \mathrm{~s}, \eta=10 \mathrm{~s}^{-1 / 2}, \delta=0,09$ e $m=0,02$; (c) $\tau=1 \times 10^{-3} \mathrm{~s}, \eta=30 \mathrm{~s}^{-1 / 2}, \delta=0,09$ e $m=0,02$; (d) $\tau=1 \times 10^{-3} \mathrm{~s}, \eta=50 \mathrm{~s}^{-1 / 2}, \delta=0,09$ e $m=0,02$; (e) $\tau=1 \times 10^{-3} \mathrm{~s}, \eta=100 \mathrm{~s}^{-1 / 2}, \delta=0,09$ e $m=0,02$; (f) $\tau=1 \times 10^{-3} \mathrm{~s}, \eta=150 \mathrm{~s}^{-1 / 2}, \delta=0,09$ e $m=0,02$.

Na Figura 8, é importante observar que para valores de $\tau=$ $10^{-6} \mathrm{~S}$, combinado com $\delta$ elevado $(\delta=0,85)$, a influência do parâmetro $\eta$ é muito pequena na fase, fazendo deslocar-se 0 seu primeiro pico desde $10^{0} \mathrm{~Hz}$ quando $\eta=3 \mathrm{~s}^{-1 / 2}$ até $10^{3} \mathrm{~Hz}$ quando $\eta=100 \mathrm{~s}^{-1 / 2}$, mas sempre com um pequeno valor (menor que $10 \mathrm{mrad}$ ), e 0 segundo pico fixo, em torno de $10^{6} \mathrm{~Hz}$, com intensidade elevada $(90 \mathrm{mrad})$. Algo semelhante ocorre com a amplitude, apresentando dois patamares com valores bai- xos muito próximos e um ramo fortemente ascendente às altas frequências, de $10^{5} \mathrm{~Hz}$ a $10^{6} \mathrm{~Hz}$.

Na Figura 9, observa-se que o parâmetro $m$, de um modo geral, é importante para a amplitude da condutividade, influenciando diretamente a assíntota desta função às frequências mais elevadas. A cargabilidade $(m)$ também atua na intensidade dos picos de fase, fazendo ambos crescerem, mas provocando uma compensação entre o primeiro e o segundo picos de fase, de 

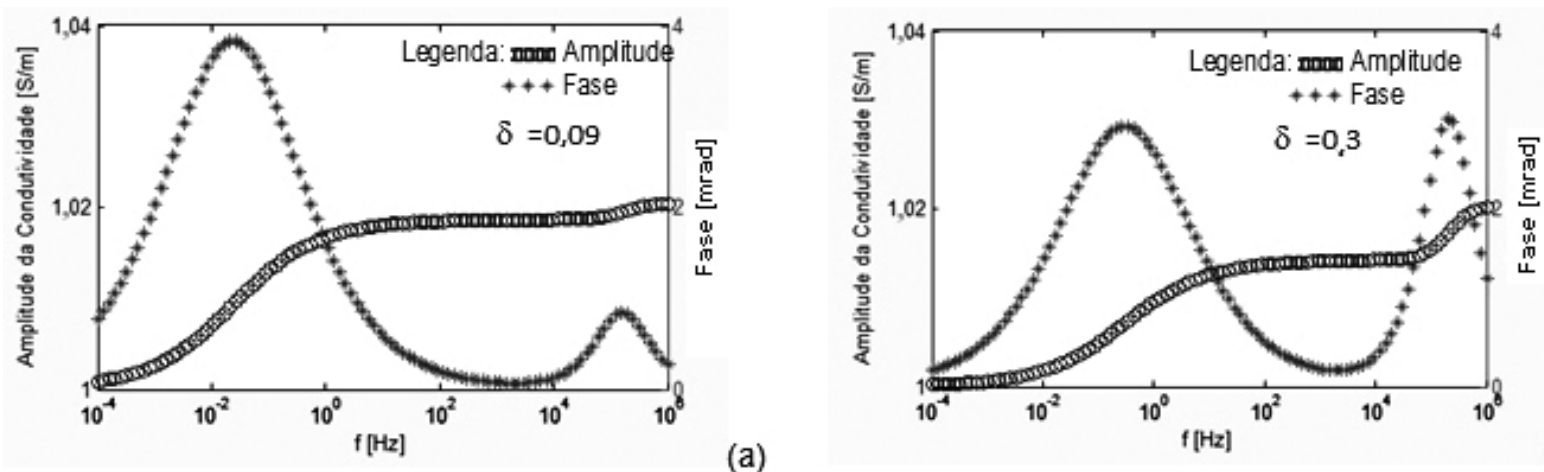

(b)
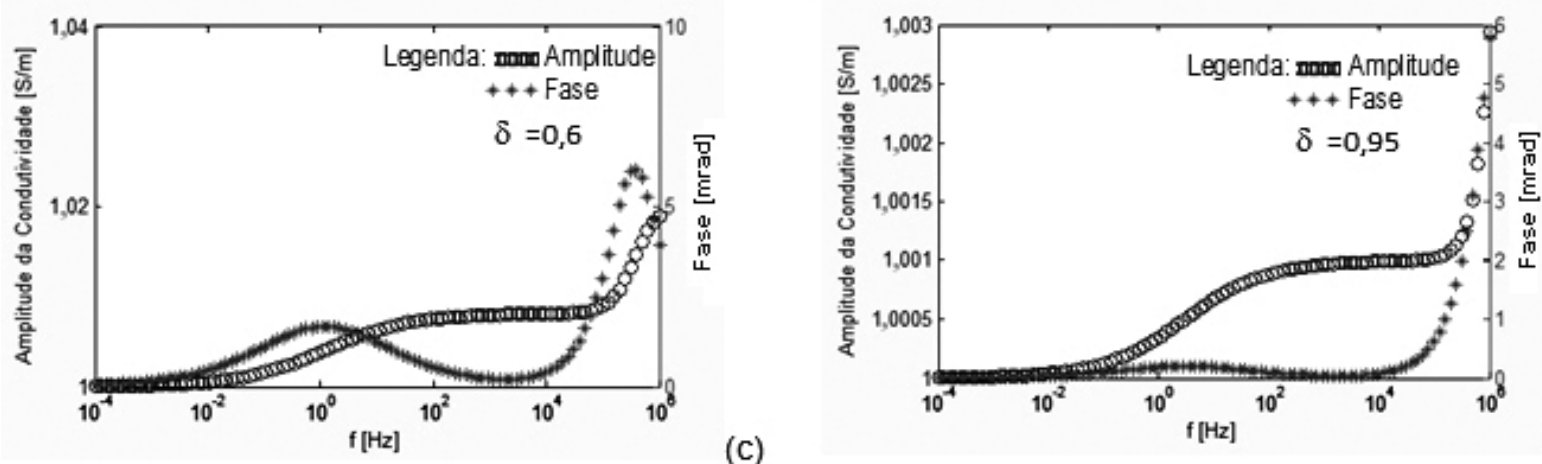

(d)

Figura 7 - Curvas de amplitude e fase da função condutividade de corrente total normalizada, com variação de $\delta$ : (a) $\tau=1 \times 10^{-6} \mathrm{~s}, \eta=4,5 \mathrm{~s}^{-1 / 2}, \delta=0,09 \mathrm{e}$ $m=0,02$; (b) $\tau=1 \times 10^{-6} \mathrm{~S}, \eta=4,5 \mathrm{~s}^{-1 / 2}, \delta=0,3$ e $m=0,02$; (c) $\tau=1 \times 10^{-6} \mathrm{~S}, \eta=4,5 \mathrm{~s}^{-1 / 2}, \delta=0,6$ e $m=0,02$; (d) $\tau=1 \times 10^{-6} \mathrm{~S}, \eta=4,5 \mathrm{~s}^{-1 / 2}$, $\delta=0,95$ e $m=0,02$.

modo contrário ao efeito produzido pelo parâmetro $\delta$ (Fig. 7), isto é, fazendo crescer 0 primeiro pico de fase em relação ao segundo pico.

Para valores intermediários de $(\eta, \tau)$, respectivamente $\left(15 \mathrm{~s}^{-1 / 2} ; 6,6 \times 10^{-5} \mathrm{~s}\right)$, quando $m$ é pequeno $(m=0,2)$, como mostra a Figura 9(a), tem-se o primeiro pico menor que 0 segundo, algo como 25 e 50 mrad. Quando $m$ é grande $(m=$ 0,95 , Fig. 9(d)), ambos os valores dos picos de fase são elevados (atingindo 450 mrad e 350 mrad, respectivamente) com um aumento do primeiro pico mais significativo comparado com 0 crescimento do segundo pico de fase.

Observando o conjunto dos gráficos analisados, nota-se que as curvas apresentam sensibilidade a pequenas variações nos parâmetros, como também, verifica-se existir forte correlação entre os pares $(\tau, \eta)$ e $(\delta, m)$. A mais notável delas relaciona-se ao par $(\tau, \eta)$, através da condição de existência de uma depressão na fase em um intervalo intermediário do espectro, determinada por Dias (2000)

$$
\eta^{2} \ll \omega \ll 1 / \tau .
$$

A condição (21), ligeiramente modificada para $\eta^{2} \tau \ll 1$, corresponde à condição de existência do segundo pico de fase.
0 fato de 0 modelo analisado poder gerar curvas com até 2 picos na fase e 3 patamares na amplitude é de grande relevância para a capacidade da função de descrever dados experimentais. Particularmente, sabendo-se que a quase totalidade dos modeIos vigentes, inclusive o "modelo Cole-Cole" mais usado atualmente, só pode gerar curvas com no máximo 1 pico na fase e 2 patamares na amplitude (Dias, 2000), não obstante existirem dados experimentais que correspondem a curvas mais caprichosas (ver, por exemplo, Mahan et al., 1986).

\section{CONCLUSÕES}

Neste trabalho, procedeu-se à análise funcional e discussão do comportamento da função "condutividade espectral" proposta por Dias (2000), tendo-se a ressaltar as seguintes características:

1) foram mostrados todos os tipos de curvas de fase e amplitude da condutividade, que o referido modelo é capaz de gerar, inclusive a influência dos valores dos seus parâmetros petrofísicos na forma das curvas foi discutida;

2) demonstrou-se que a função "condutividade espectral" desse modelo tem a capacidade de poder exibir até 2 

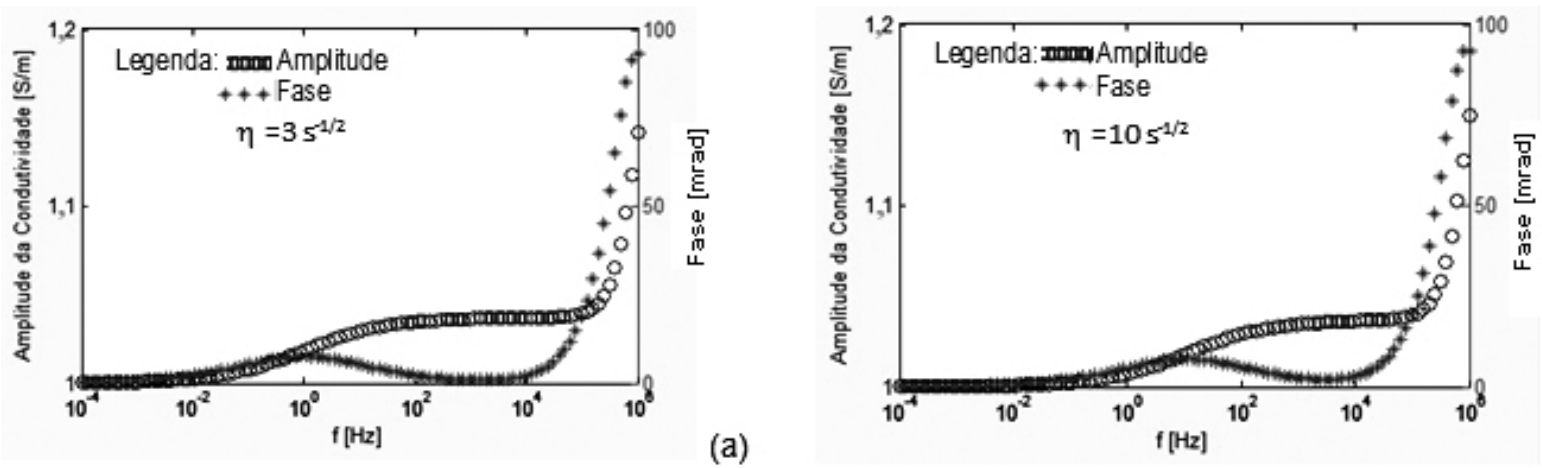

(b)
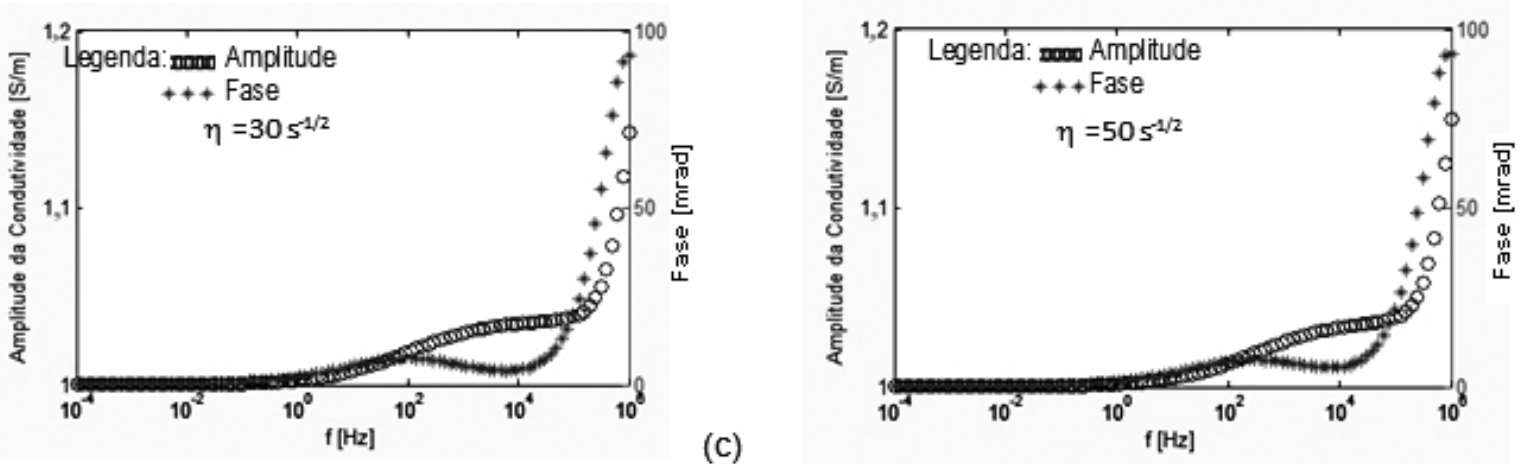

(d)
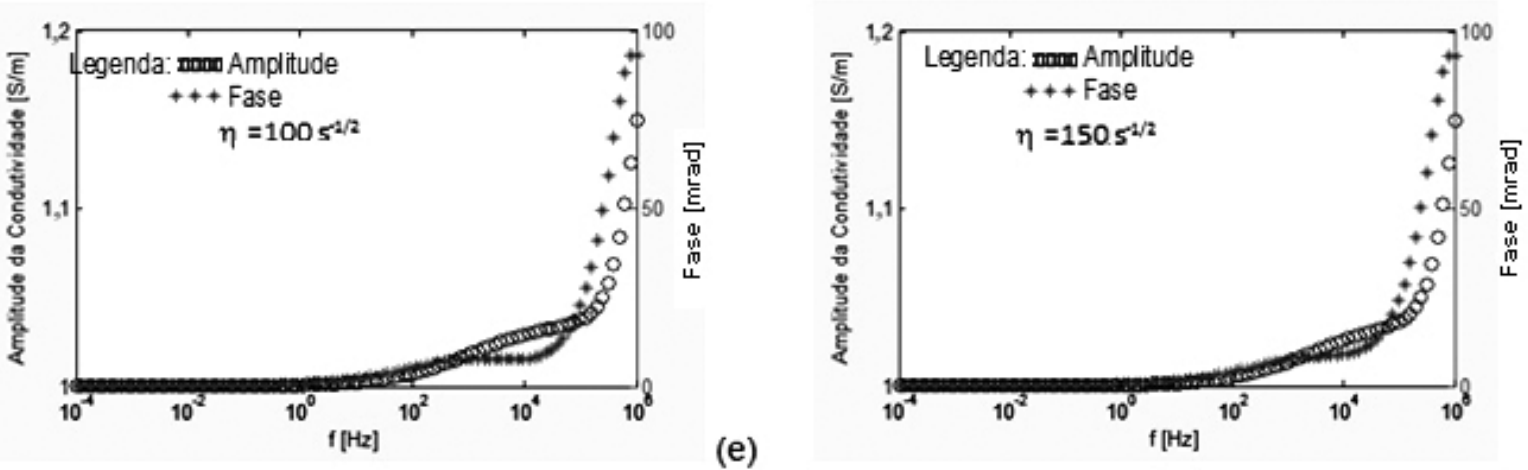

(f)

Figura 8 - Curvas de amplitude e fase da função condutividade de corrente total normalizada, com variação de $\eta$ sendo $\tau$ pequeno $\left(10^{-6}\right.$ s) e $\delta$ grande $(0,85)$ : (a) $\tau=1 \times 10^{-6} \mathrm{~S}, \eta=3 \mathrm{~s}^{-1 / 2}, \delta=0,85$ e $m=0,2$; (b) $\tau=1 \times 10^{-6} \mathrm{~S}, \eta=10 \mathrm{~s}^{-1 / 2}, \delta=0,85$ e $m=0,2$; (c) $\tau=1 \times 10^{-6} \mathrm{~s}, \eta=30 \mathrm{~s}^{-1 / 2}, \delta=0,85 \mathrm{e}$ $m=0,2$; (d) $\tau=1 \times 10-6 \mathrm{~s}, \eta=50 \mathrm{~s}^{-1 / 2}, \delta=0,85$ e $m=0,2$; (e) $\tau=1 \times 10^{-6} \mathrm{~s}, \eta=100 \mathrm{~s}^{-1 / 2}, \delta=0,85$ e $m=0,2$; (f) $\tau=1 \times 10^{-6} \mathrm{~s}, \eta=150 \mathrm{~s}^{-1 / 2}$, $\delta=0,85$ e $m=0,2$

picos na fase e 3 patamares na amplitude da condutividade. Esse fato the confere uma singular flexibilidade para descrever dados experimentais sobre o fenômeno IP (considerando-se que a quase totalidade dos modelos em uso geram funções com no máximo 1 pico na fase e 2 patamares na amplitude);

3) verificou-se existir forte correlação entre os pares dos parâmetros petrofísicos $(\tau, \eta)$ e $(\delta, m)$. Existe uma in- fluência mais forte entre os parâmetros $(\tau, \eta)$, revelada na posição dos picos de fase, inclusive sendo possível estabelecer a condição de existência do segundo pico, dada por $\eta^{2} \tau \ll 1$;

4) verificou-se haver maior sensibilidade da função aos valores pequenos a médios do parâmetro $\eta$, isto é, para $\eta<30 \mathrm{~s}^{-1 / 2}$. 


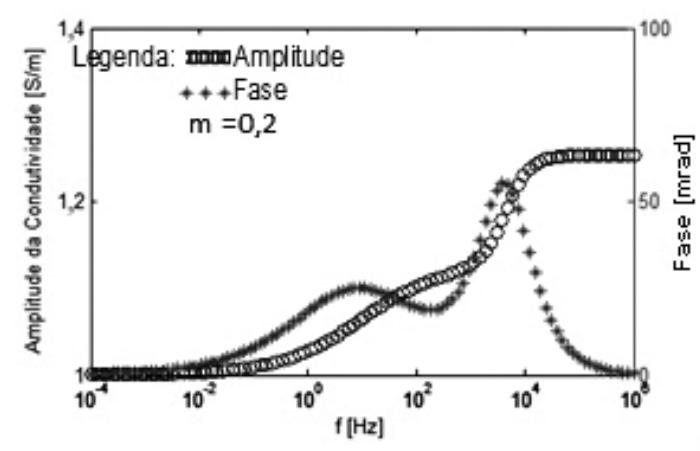

(a)

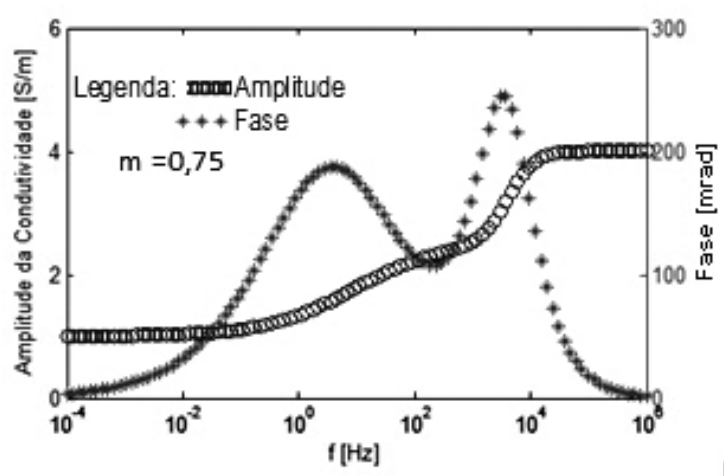

(c)

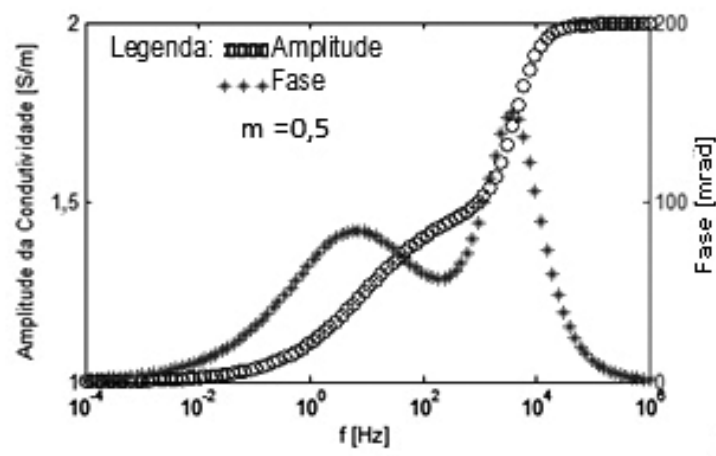

(b)

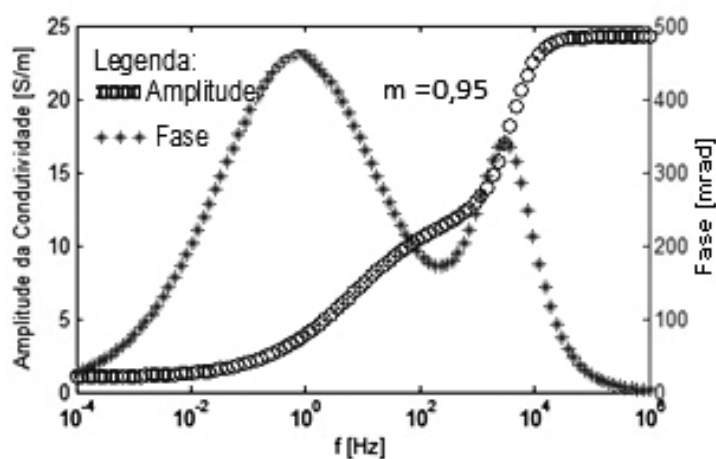

(d)

Figura 9 - Curvas de amplitude e fase da função condutividade de corrente total normalizada, com variação de $m$ : (a) $\tau=6,6 \times 10^{-5} \mathrm{~s}, \eta=15 \mathrm{~s}^{-1 / 2}, \delta=0,5 \mathrm{e}$ $m=0,2$; (b) $\tau=6,6 \times 10^{-5} \mathrm{~s}, \eta=15 \mathrm{~s}^{-1 / 2}, \delta=0,5$ e $m=0,5$; (c) $\tau=6,6 \times 10^{-5} \mathrm{~s}, \eta=15 \mathrm{~s}^{-1 / 2}, \delta=0,5$ e $m=0,75$; (d) $\tau=6,6 \times 10^{-5} \mathrm{~s}, \eta=15 \mathrm{~s}^{-1 / 2}$, $\delta=0,5$ e $m=0,95$.

\section{APÊNDICE}

\section{TRANSFORMAÇÃO ALGÉBRICA DA EQUAÇÃO (3) NA (4)}

Inicia-se com a função

$$
\frac{R}{R_{S}+\frac{\left(r+Z_{w}\right) Z_{C_{d l}}}{r+Z_{w}+Z_{C_{d l}}}}=\frac{R}{D}
$$

e procede-se à seguinte transformação algébrica na expressão do denominador $D$ :

$$
\begin{aligned}
& D=R_{S}+\frac{\left(r+Z_{w}\right) Z_{C_{d l}}}{r+Z_{w}+Z_{C_{d l}}}=R_{S}+\frac{\left(1+r / Z_{w}\right) Z_{w}}{1+\left(r+Z_{w}\right) / Z_{C_{d l}}} \\
& D=\frac{1+r / Z_{w}+R_{S}\left(1+\left(r+Z_{w}\right) / Z_{C_{d l}}\right) / Z_{w}}{\left(1+\left(r+Z_{w}\right) / Z_{C_{d l}}\right) / Z_{w}} \\
& D=\frac{1+\left[r+R_{s}+R_{S}\left(r+Z_{w}\right) / Z_{C_{d l}}\right] / Z_{w}}{\left(1+\left(r+Z_{w}\right) / Z_{C_{d l}}\right) / Z_{w}} \\
& D=\frac{1+\left(r+R_{S}\right)\left[1+\left(R_{S} /\left(r+R_{S}\right)\right)\left(r+Z_{w}\right) / Z_{C_{d l}}\right] / Z_{w}}{\left(1+\left(r+Z_{w}\right) / Z_{C_{d l}}\right) / Z_{w}} \\
& D=\frac{1+\left(r+R_{S}\right)\left[1+\left(1-r /\left(r+R_{S}\right)\right)\left(r+Z_{w}\right) / Z_{C_{d l}}\right] / Z_{w}}{\left(1+\left(r+Z_{w}\right) / Z_{C_{d l}}\right) / Z_{w}}
\end{aligned}
$$


Portanto, (A.1) pode ser reescrita como

$$
\frac{R}{R_{S}+\frac{\left(r+Z_{w}\right) Z_{C_{d l}}}{r+Z_{w}+Z_{C_{d l}}}}=\frac{R}{D}=\frac{R\left(1+\left(r+Z_{w}\right) / Z_{C_{d l}}\right) / Z_{w}}{1+\left(r+R_{S}\right)\left[1+\left(1-r /\left(r+R_{S}\right)\right)\left(r+Z_{w}\right) / Z_{C_{d l}}\right] / Z_{w}} .
$$

\section{AGRADECIMENTOS}

Os autores agradecem as observações e comentários feitos pelo revisor anônimo, que contribuíram para melhorar a qualidade deste trabalho. Também agradecem à FAPERJ, pela bolsa de mestrado ao primeiro autor, bem como pelo Grant "Cientista do Nosso Estado" / FAPERJ e pela bolsa da CAPES no Programa PVNS / UFPA ao segundo autor, para realização desta pesquisa.

\section{REFERÊNCIAS}

BAMBERG A, COCKBURN B, GOLDMAN Y, JOLY P \& KERN M. 1989. Numerical solution of Maxwell's equations in a conductive and polarizable medium. Computer Methods in Applied Mechanics and Engineering, 75: 11-25.

BOCKRIS JO \& REDDY AKN. 1970. Modern Electrochemistry. Plenum Press, New York, NY, $1432 \mathrm{p}$.

BRANT AA. 1959. Historical summary of overvoltage developments by Newmont Exploration Limited 1946-1955. In: WAIT JR (Ed.). Overvoltage Research and Geophysical Applications. Pergamon Press, Internat. Series on Earth Sciences, 4: 1-3.

CHANG HC \& JAFFÉ G. 1952. Polarization in electrolytic solutions. Part I: Theory. J. Chem. Phys., 20(7): 1071-1077.

COCKBURN B. 1983. Étude mathématique et numérique des équations de Maxwell dans des milieux polarisables. DSc. Thèse. Université Paris IX, France, $278 \mathrm{p}$.

COLE KS \& COLE RH. 1941. Dispersion and absorption in dielectrics I: Alternating current characteristics. Journal of Chemical Physics, 9: 341-351.

DEBYE P. 1929. Polar Molecules. Dover Publications, New York, NY, $171 p$.

DIAS CA. 1968. A non-grounded method for measuring induced electrical polarization and conductivity. Ph.D. Thesis. University of California, Berkeley, USA. AAT 6914871. $272 \mathrm{p}$.

DIAS CA. 1972. An analytical model for a polarizable medium at radio and lower frequencies. Journal of Geophysical Research, 77(26): 49454956.

DIAS CA. 2000. Developments in a model to describe low-frequency electrical polarization of rocks. Geophysics, 65(2): 437-451.

DIAS CA, SATO HK \& LIMA OAL. 2005. Multi-frequency EM method for hydrocarbon detection and for monitoring fluid invasion during enhanced oil recovery. In: Expanded Abstract, Paper \#EMP 1.2, 602-604,
CD-ROM, $75^{\text {th }}$ Annual International SEG Meeting, Nov., 6-11, Houston, Texas.

DIAS CA, LIMA OAL, SATO HK \& MORAES JAC. 2006. Contribution to oil exploration and development - A successful inductive multifrequency EM survey on-shore Brazil. In: Expanded Abstract, 4 p., CDROM, $68^{\text {th }}$ EAGE Conference \& Exhibition, Jun. 12-15, Vienna, Austria.

FAINBERG EB, BARSUKOV P \& KJERSTAD JK. 2009. Method and device for induced polarization mapping of submarine hydrocarbon reservoirs. International Patent W0 2009/082236 A1, WIPO/International Bureau, $38 \mathrm{p}$.

GRAHAME DC. 1952. Mathematical theory of the faradaic admittance (pseudocapacity and polarization resistance). J. Electrochem. Soc., 99: 370c-385c.

HASHSISH EA. 2002. Detection of polarizable media of a multilayered earth model. Journal of Electromagnetic Waves and Applications, 16(8): $1119-1134$.

LIMA OAL. 1995. Water saturation and permeability from resistivity, dielectric, and porosity logs. Geophysics, 60(6): 1756-1764.

LIMA OAL \& NIWAS S. 2000. Estimation of hydraulic parameters of shaly sandstone aquifers from geoelectrical measurements. Journal of Hydrology, 235: 12-26.

LIMA OAL, CLENNELL MB, NERY GG \& NIWAS S. 2005. A volumetric approach for the resistivity response of freshwater shaly sandstones. Geophysics, 70(1): F1-F10.

MADDEN TR \& MARSHALL DJ. 1959a. Electrode and Membrane Polarization. MIT Report to US Atomic Commission, Vols. RME-3157.

MADDEN TR \& MARSHALL DJ. 1959b. Induced polarization - A study of its causes and magnitudes in geologic materials. MIT Report to US Atomic Commission, Vols. RME-3169.

MAHAN MK, REDMAN JD \& STRANGWAY DW. 1986. Complex resistivity of synthetic sulfide bearing rocks. Geophysical Prospecting, 34: 743-768.

MAOSONG T \& HONGGEN T. 2008. Permeability estimating from complex resistivity measurement of shaly sand reservoir. Geophysical Journal International, 173(2): 733-739.

MAOSONG T, WEINAN W, YIZHONG J, DEQIN S \& LI L. 2004. Estimation of permeability of shaly sand reservoir from induced polarization time spectra. Journal of Petroleum Science and Engineering, 45: 1-10. 
MAOSONG T, WEINAN W, YIZHONG J \& LI L. 2006a. A time-domain induced-polarization method for estimating permeability in a shaly sand reservoir. Geophysical Prospecting, 54: 623-631.

MAOSONG T, WEINAN W, YIZHONG J, DEQIN S \& LI L. 2006b. Determining capillary-pressure curve, pore-size distribution, and permeability from induced polarization of shaly sand. Geophysics, 71(3): N33-N40.

MORRISON HF, PHILLIPS RJ \& O'BRIEN DP. 1969. Quantitative interpretation of transient electromagnetic fields over a layered half-space. Geophysical Prospecting, 17(1): 82-101.

OLHOEFT GR. 1985. Low frequency electrical properties. Geophysics, 50: 2492-2503.

PANOFSKY WKH \& PHILLIPS M. 2004. Classical Electricity and Magnetism. $2^{\text {nd }}$ edition, Dover Publications. $512 \mathrm{p}$.

PELTON WH, WARD SH, HALLOF PG, SILL WR \& NELSON PH. 1978. Mineral discrimination and removal of inductive coupling with multifrequency IP. Geophysics, 43: 588-609.

SIEGEL HO. 1959. Mathematical formulation and type curves for induced polarization. Geophysics, 24: 547-565.
WAIT JR. 1959a. A phenomenological theory of overvoltage for metallic particles. In: WAIT JR (Ed.). Overvoltage Research and Geophysical Applications. Pergamon Press, Internat. Series on Earth Sciences, 4: 22-28.

WAIT JR. 1959b. The variable-frequency method. In: WAIT JR (Ed.). Overvoltage Research and Geophysical Applications. Pergamon Press, Internat. Series on Earth Sciences, 4: 29-49.

WARD SH \& FRASER DC. 1967. Conduction of electricity in rocks. In: Society of Exploration Geophysicists. Ed. Comm., Mining Geophysics, II: 197-223.

XUSAN C, WENJIE Z \& LIUFANG Z. 2001. Complex resistivity logging and its applications. (Revista chinesa) Well Logging Technology, 25(5): 327-331.

YUE A-P, DI Q-Y, WANG M-Y \& SHI K-F. 2009. 1-D forward modeling of the CSAMT signal incorporating IP effect. (Revista chinesa) Chinese Journal of Geophysics (Acta Geophysica Sinica), 52(7): 1937-1946.

ZHU D, MAOSONG T \& TAO P. 2005. The Dias model of rock complex resistivity and its parameter inversion method. (Revista chinesa) Petroleum Geology \& Oilfield Development in Daqin, 24(5): 90-92.

\section{NOTAS SOBRE OS AUTORES}

Edivagner da Silva Ribeiro. Licenciado em Física (2007) pela Universidade Federal do Mato Grosso e Mestre em Engenharia de Reservatório e Exploração (2010) pelo LENEP/UENF. Tem como principais áreas de interesse geofísica aplicada e petrofísica.

Carlos Alberto Dias. B.Sc. em Física pelo CBPF/FNFi - ex-Univ. do Brasil (1961), M.Sc. (1965) e Ph.D. (1968) em Eng. Science / Geophysics - University of California - Berkeley / USA. Professor titular aposentado da UFPA e da UENF. Fundador e sócio da Sociedade Brasileira de Geofísica. Tem experiência em pesquisa, ensino e formação de quadros nas áreas de Geofísica Aplicada e Eng. de E\&P de Petróleo. Tem atuado em medida laboratorial e teoria da condutividade espectral e medida eletromagnética da resistividade e do efeito IP, no campo. Ultimamente, dedica-se à E\&P de petróleo e à caracterização de reservatórios usando 0 método eletromagnético a multifrequência, de sua invenção. 